\title{
The increasing significance of impact within the Research Excellence Framework (REF)
}

\section{Abstract}

Introduction

The UK's Research Excellence Framework (REF) is a performance-based research funding system that was introduced in 2014. REF is a process of expert review in universities which assesses the quality and reach of research across higher education. It highlights areas of excellence, benchmarks the quality of previously funded research and provides accountability for ongoing public investment in research. In addition to assessing the quality of research outputs and the environment in which research is undertaken, the REF became the first exercise to evaluate the impact of research outside of academia.

\section{Discussion}

This article specifically addresses the impact component of the REF, explaining what is understood by the term 'impact' and considering how impact is gaining significance within the REF framework as a measure of research excellence. It also reflects on the complexities of measuring impact and ongoing resistance from academics to the impact agenda, which they believe threatens academic freedom and appears only to value research that generates specific outcomes. In order to address these and other issues arising from the first-ever assessment of impact in REF 2014, institutions were consulted on the proposed guidance for the REF 2021 submission.

\section{Conclusion}

The final REF 2021 guidance has sought to provide clearer detail on interpretations of impact and its underpinning research in order to reassure researchers that their academic freedom will not come under threat. However, this process still fails to capture other impactful research being undertaken across institutions, which falls outside of the strict REF criteria.

\section{$\underline{\text { Key words }}$}

Research Excellence Framework, REF, research impact, accountability. 


\section{Introduction}

Within the UK, the Research Excellence Framework (REF) is a performance-based research funding system that was introduced for the first time in 2014 as a successor to the Research Assessment Exercise (RAE). The first RAE was undertaken in 1986 to provide an explicit and formalised assessment process of the quality of research, with subsequent exercises taking place until 2008. REF is a process of expert review in higher education institutions (HEIs) / universities which assesses the quality and reach of research across UK higher education, seeking to highlight areas of excellence, whilst benchmarking the quality of previously funded research and providing accountability for ongoing public investment in research. The four UK higher education funding bodies (Research England; Scottish Funding Council; Higher Education Funding Council for Wales; Department for the Economy, Northern Ireland) are jointly responsible for the undertaking of the REF exercise. ${ }^{1}$

In addition to assessing the quality of outputs (e.g. journal papers) and the environment in which research is carried out, the REF became the first exercise to evaluate the impact of research outside of academia. UK HEls submitted some 6,975 impact case studies to showcase the real-world impact of their research for REF $2014 .^{2}$ In light of this, the REF is perceived by commentators, such as Watermeyer, as being a game-changer for researchers in HEls in terms of the way in which they conceptualise and conduct their research and also how this challenges perceptions about what is considered excellence in research. ${ }^{3}$

The purpose of this article is to address the impact component of the REF, firstly by explaining what is understood by the term 'impact' in this context and subsequently by considering how impact is growing in importance within the framework of the REF as a measure of research excellence. The article concludes by reflecting on the complexities of measuring impact within the confines of a prescriptive case study format.

\section{Definition of impact}


UK Research and Innovation (UKRI) works in partnership with universities and other organisations to enable the UK's research environment to flourish. UKRI invests some $f 7$ billion annually in research and innovation and makes a clear distinction between academic impact (i.e. the contribution that research makes within the academy) and economic and societal impact (i.e. the demonstrable contribution that excellent research makes to society and the economy). ${ }^{4}$ Examples of impact include: creation and sharing of new knowledge and innovation; invention of ground-breaking products, companies and jobs; enhancement of quality of life and health. The reason for making this distinction is that economic and societal impact provides a level of accountability for the spending of public money; it improves the quality of research because it enables engagement with a wide range of beneficiaries and enhances the UK's reputation as a place to invest in research and innovation.

Similarly, Research England, which oversees the UKRI's functions with respect to research and knowledge exchange in English universities, defines impact as: "an effect on, change or benefit to the economy, society, culture, public policy or services, health, the environment or quality of life, beyond academia", measuring impact within the REF in terms of both reach (the extent and/or diversity of the beneficiaries of the impact, as relevant to the nature of the impact) and significance (the degree to which the impact has enabled, enriched, influenced, informed or changed the performance, policies, practices, products, services, understanding, awareness or well-being of the beneficiaries). ${ }^{2}$

\section{The increasing importance of impact within the REF}

Based on REF 2014 data, a 4* impact case study is estimated to be worth around $f 324,000$ to an institution in quality-related research (QR) funding over the course of the REF cycle (67 years on average). ${ }^{5} \mathrm{~A}$ high-quality impact case study represents the equivalent value of up to 7 high-quality research outputs. ${ }^{3}$ Following Lord Stern's independent review of university research funding in 2016 and the policy commitment to impact in future REF exercises, ${ }^{6}$ the decision was taken by the Higher Education Funding Council for England (superseded by UKRI in April 2018) to increase the weighting for impact from $20 \%$ of the overall total in REF 
2014 to $25 \%$ in REF $2021,{ }^{7}$ which would appear to indicate that each top-scoring impact case study will be worth more money to each institution in the next cycle of funding (from 2021 onwards).

In determining the weightings for the REF exercise, three distinct components are evaluated, namely research outputs, impact and the environment, in order to obtain an overall quality profile for each institution. ${ }^{7}$ The weightings are as follows:

\begin{tabular}{|l|l|l|}
\hline Component & Form of assessment & $\begin{array}{l}\text { Weighting as \% of } \\
\text { overall score }\end{array}$ \\
\hline $\begin{array}{l}\text { Outputs (journal articles, books, reports, } \\
\text { conference contributions etc.) }\end{array}$ & $\begin{array}{l}\text { Quality of research in terms of } \\
\text { originality, significance and rigour }\end{array}$ & $\mathbf{6 0 \%}$ \\
\hline $\begin{array}{l}\text { Impact (an effect, change or benefit } \\
\text { beyond academia) }\end{array}$ & $\begin{array}{l}\text { Reach and significance of impact } \\
\text { underpinned by excellent research }\end{array}$ & $\mathbf{2 5 \%}$ \\
\hline $\begin{array}{l}\text { Environment (research strategy, people, } \\
\text { income, infrastructure, collaborations) }\end{array}$ & $\begin{array}{l}\text { Contribution to the vitality and } \\
\text { sustainability of the research base }\end{array}$ & $\mathbf{1 5 \%}$ \\
\hline
\end{tabular}

An institution is awarded QR funding according to the overall quality profile.

The UK's higher education sector has become increasingly characterised by its audit culture and the requirement to make researchers more accountable, as the recipients of public funds. ${ }^{3}$ This explains the rationale behind the increased impact weighting within the REF exercise and also the growing trend among external funders, for example, research councils, to require applicants to include pathways to impact and impact statements within funding applications in order to demonstrate a level of accountability for publicly-funded research that will have a tangible value and benefit beyond the world of academia. As such, researchers are increasingly having to rationalise their research as a public investment. ${ }^{8}$ By including stakeholders and publics in the research process there is automatically greater buy-in and a higher likelihood of achieving benefit and change for these groups as a result of the research.

Researchers are being challenged to change the way in which they think about their research in order to demonstrate their 'impactfulness' through inclusion of a non-academic 
impact element. ${ }^{8}$ Indeed, it is becoming commonplace to see funded research projects that cost in an 'impact officer' as an additional resource to help with impact data collection and analysis throughout the project lifecycle. In order to support the impact process further, HEIs have begun revising their ethics approval processes to ensure that impact capture and collection is considered from the outset of projects. Researchers must therefore give due consideration to the impact outcomes of their research at an early stage of each project and this enables them to collect evidence both during and after completion of the project in order to demonstrate change.

The growing trend towards the showcasing of real-world research impact and the huge cost implications of generating a 4* REF impact case study has likewise challenged institutions to provide additional support to researchers to help them to realise their impact and to create stellar case studies of the future. This ranges from the offer of seedcorn funding and other internal impact funds to support research projects with high impact potential, the creation of additional impact officer posts to assist with case study development, impact and public engagement training and workshops, use of academic 'impact champions' to mentor colleagues in the development of research proposals, or simply signposting to a variety of free online impact resources to help build impact. ${ }^{10}$

\section{Addressing resistance to the reporting of impact}

Commentators suggest that there has been a history of resistance to the impact agenda, with academics railing against a performance-related system of funding allocation which serves to stifle creativity, threaten academic freedom and which seemingly places greatest value on research that generates specific outcomes. ${ }^{8}$ This is not least due to the numerous shortcomings identified when assessing the quality of research impact in REF 2014: for example, a major drawback was that the artificial nature of the REF timeframe made measurement of impact somewhat imprecise. Other issues included the following: not all institutions put in place sufficient infrastructure to allow for impact generation; academics did not as a rule record and evaluate their impact activities; research activity tended to be compartmentalised into the 'best' case studies which was not truly indicative of the broad- 
ranging activity taking place; the line of causality between research and impact, for example on policymaking, was often speculative; some research areas did not lend themselves so easily to impact, for example, less applied fields such as natural science; some impacts were difficult to evidence, making attribution complex, for example, in the arts and humanities fields; the importance of public engagement was downplayed, relegating it to a 'pathway to impact' that was seen by many as non-essential..$^{8,9}$ The list goes on.

Although recent research suggests that pursuit of impact does not appear to have had a detrimental effect on the quality of research, ${ }^{9}$ it was acknowledged through the Stern Review and subsequent consultation process that many of the concerns raised by academics and institutions about the first-ever assessment of impact in REF 2014 were indeed valid and institutions were therefore able to feed back on the proposed guidance for the REF 2021 submission. ${ }^{6}$ The final guidance sought to provide clearer detail on interpretations of impact and its underpinning research in order to reassure researchers that their academic freedom would not being curtailed by the process. ${ }^{9}$ For example, the REF 2021 Panel Criteria and Working Methods guidance ${ }^{7}$ makes clear that the body of outputs as a whole will be assessed in terms of meeting the $2^{*}$ quality threshold, allowing greater freedom to submit 'non-traditional' outputs as part of this evidence; it allows for continued case studies from REF 2014 to be submitted, acknowledging the fact that impact often takes many years to develop and does not fit neatly into a rigid timeframe; it increasingly recognises the role of public engagement in research and how it can be applied to achieve impact. A list of examples of impact and indicators is also provided by way of demonstrating the wideranging types of impact that can be used within a case study, stressing that the relationship between research and impact is understood to be indirect or non-linear (for example, coproduced research impacts) in many cases. It is clear that for this second iteration of REF impact institutions also now have a deeper understanding of what is required in terms of time and money resource to aid development of an impact case study over a period of years and appropriate infrastructure has been put into place to support this.

It is also worthy of note that impact forms a core part of the environment narrative (environment weighting is $15 \%$ of the overall total, with around $4 \%$ of this $15 \%$ being allocated to impact), with institutions being required to outline an impact strategy around Page $6 / 7$ 
how they are currently supporting impact generation and capture, as well as identifying how they propose to continue supporting impact in the future.

This is not to suggest, however, that only research that is included as part of a REF impact case study is considered to be impactful. There are many examples of research that takes place at a local level and on a smaller scale which deliver enormous benefit to a community, such as the creation of the Salford Anti-Poverty Taskforce, which is a joint University of Salford and Salford Council project carrying out research into the lived experiences of people in poverty in order to inform future policy, and the delivery of a range of Knowledge Transfer Partnerships between the University of Salford and organisations such as Greater Manchester Fire and Rescue Service, as well as local enterprises, which generate commercial and social impact for these stakeholders, but which may not have the necessary reach (i.e. extent and/or diversity of beneficiaries) to perform well in REF or which may not have led to a published REFable output, therefore deeming the impact ineligible for a case study submission. ${ }^{11}$

\section{Conclusion}

While it is clear that the demonstration of real-world impact is fast becoming embedded within research activity, there still appears to be resistance within academia to its growing pervasiveness and an underlying resentment that 'doing impact' is simply another box to tick. The REF exercise is a means of increasing the reporting around impact activities, albeit with restrictions and constraints, and is pivotal to institutions in determining the distribution of QR money that can be used to invest in future research activities. However, what it does not capture is the wealth of other exciting and impactful research being undertaken across our institutions, which falls outside of the margins of eligibility for the REF, but which is no less valuable to the beneficiaries of the research or to the researchers who are seeking to give something back at a local level, for example.

Word count: 2118

Declarations of interest: none 


\section{References}

1. REF2021 Research Excellence Framework. What is the REF? Available at: https://www.ref.ac.uk/about/what-is-the-ref/. [Accessed 20 December 2019]

2. Research England. REF impact. Available at: https://re.ukri.org/research/ref-impact/ [Accessed 20 December 2019]

3. Watermeyer R, Chubb J. Evaluating 'impact' in the UK's Research Excellence Framework (REF): liminality, looseness and new modalities of scholarly distinction. Studies in Higher Education 2019; 44:9, 1554-1566. doi: 10.1080/03075079.2018.1455082.

4. UK Research and Innovation. Excellence with impact. Available at: https://www.ukri.org/innovation/excellence-with-impact/ [Accessed 20 December 2019]

5. Reed, M, Kerridge, S. Fast Track Impact. How much was an impact case study worth in the Research Excellence Framework. 1 February 2017. Available at:

https://www.fasttrackimpact.com/single-post/2017/02/01/How-much-was-an-impact-casestudy-worth-in-the-UK-Research-Excellence-Framework [Accessed 20 December 2019] 6. Research Excellence Framework Review. Available at: https://www.gov.uk/government/publications/research-excellence-framework-review [Accessed 20 December 2019]

7. REF2021 Research Excellence Framework. Panel Criteria and Working Methods (2019/02). Available at: https://www.ref.ac.uk/media/1084/ref-2019 02-panel-criteria-and-workingmethods.pdf. January 2019. [Accessed 20 December 2019]

8. Watermeyer R. Impact in the REF: issues and obstacles. Studies in Higher Education 2016; 41:2, 199-214. doi: 10.1080/03075079.2014.915303.

9. Terama E, Smallman M, Lock SJ, Johnson C, Austwick MZ. Beyond academia interrogating research impact in the Research Excellence Framework. PLoS ONE 2016; 11(12). doi: 10.1371/journal.pone.0168533.

10. Fast Track Impact. Resources. Available at: https://www.fasttrackimpact.com/resources [Accessed 14 February 2020]

11. University of Salford. Social impact. Available at: https://www.salford.ac.uk/salforduntold [Accessed 14 February 2020] 